\title{
Analysis of the Transient Dynamics of Coupled-Oscillator Systems
}

\author{
Sergio Sancho, Almudena Suarez, Franco Ramirez \\ Department of Communications Engineering, University of Cantabria, Spain
}

\begin{abstract}
A realistic reduced-order formulation of systems containing several transistor-based oscillators, such as coupledoscillator networks, is presented. The formulation is able to predict the oscillation build-up and other transient effects for the first time to our knowledge. The individual oscillator models are constructed from a nonlinear admittance function extracted from circuit-level harmonic-balance simulations. These models are used to derive a nonlinear differential-equation system able to describe the transient behavior of the entire structure. For illustration, the method has been applied to a coupled-oscillator system at $5 \mathrm{GHz}$, obtaining very good agreement with circuit-level envelope transient (when applicable) and with measurements.
\end{abstract}

Keywords-coupled-oscillator systems, transient analysis.

\section{INTRODUCTION}

Systems containing oscillator circuits, whose operation may be affected by other system components [1]-[7], are difficult to simulate since their oscillation frequency, and even their stability properties, may change under the influence of these components. One example of this complexity is found in coupled-oscillator systems, used for power-combination and beam steering [2]-[7]. Their harmonic-balance (HB) simulation requires the individual fulfillment of the oscillation condition in each oscillator circuit, which can be achieved through the use of one auxiliary generator (AG) per oscillator element [8]. However, and due to the lack of symmetry in the coupled system, convergence failures arise from a certain number of oscillator elements [9]. In order to cope with this problem, several previous works propose the use of oscillator models extracted from a HB simulation of the standalone oscillator circuit [9]-[11]. These models are based on the linearization of an oscillator admittance function $Y$ about the free-running solution. This solution fulfills $Y=0$ and the linearization is carried out with the aid of a voltage-type auxiliary generator (AG), applying finite differences to the amplitude $(V)$ and frequency $(\omega)$ of the AG [12]. In comparison with the commonly used Van der Pol models, this method has the advantage of enabling a realistic prediction of the behavior of practical systems of transistor-based oscillators. However, because the HB models are extracted linearizing the oscillator circuit about its free-running solution, they are only valid under weak coupling conditions. Furthermore, they are unable to predict the oscillation build-up due to the large amplitude excursion of the start-up transient.

The recent works [13], [14] present a methodology to extract a reduced-order nonlinear model of a single oscillator, which is able to predict the build-up transient. The model relies on the application of the implicit function theorem to the HB system, in order to derive a reduced-order nonlinear differential equation at the observation node. One should emphasize that unlike what is done in the models in [9], [10], [15], this admittance function must be a nonlinear one, able to account for the large-amplitude excursion during the oscillation startup. The nonlinear function $Y(V, \omega)$ is extracted through a double sweep in the excitation amplitude $V$ and frequency $\omega$ of an AG, performing a HB simulation at each sweep step. Once the bi-variate nonlinear admittance function $Y(V, \omega)$ is available, the dynamic oscillator model is obtained by locally linearizing this function about each transient amplitude $V$, which, as the amplitude evolves, provides a sequence of linear ordinary differential equations describing the system dynamics.

So far, the reduced-order oscillator model has enabled the prediction of the transient behavior of a single oscillator circuit in a computationally efficient manner. However, this kind of model will be most useful when applied to complex systems, such as coupled-oscillator networks, phase locked-loops, and any system in which an oscillator is switched on and off. This work proposes a new a methodology to analyze complex oscillator systems through a nonlinear differential-equation system based on the described reduced-order models of the oscillator elements. For illustration, the method will be applied to a system of three coupled oscillators at $5 \mathrm{GHz}$, which has been implemented and measured.

\section{TIME-FREQUENCY DOMAIN OF A COUPLED-OSCILLATOR SYSTEM}

As described in [9], [10], applying the implicit function theorem, the HB system of equations associated with a given free-running oscillator can be reduced to a single complex equation. This is the first harmonic equation of the Kirchhoff current law $(\mathrm{KCL})$ at a particular observation node $q: I(V, \omega)=$ $Y(V, \omega) V e^{j \phi}=0$, where $V$ and $\phi$ are the first harmonic amplitude and phase of the voltage signal at the node $q, \omega$ is the free-running frequency, and $Y(V, \omega)$ is the admittance function at $q$, calculated at the first harmonic.

In the case of a system of $N$ coupled oscillators, assumed different, one function $Y_{i}\left(V_{i}, \omega_{i}\right)$ will be derived for each oscillator element, applying a double sweep in the AG amplitude $V_{i}$ and frequency $\omega_{i}$, and performing a $\mathrm{HB}$ simulation at each sweep step. In each case, the observation node $q_{i}$ corresponds to the one at which the oscillator is connected to the coupling network. To derive the differentialequation system, one will consider first the steady-state system in a locked condition at the fundamental frequency $\omega$ :

$$
\begin{aligned}
& Y_{i}\left(V_{i}, \omega\right) X_{i}+\sum_{k=1}^{N} Y_{i k}^{c}(\omega) X_{k}=0, \\
& i=1, \ldots, N
\end{aligned}
$$


where $X_{i} \equiv V_{i} e^{j \phi_{i}}$ is the first harmonic of the voltage signal of each $i$-th oscillator at the observation node and $Y_{i k}^{c}(\omega)$ are the components of the admittance matrix of the linear coupling network. System (1) will be fulfilled in a locked condition at the frequency $\omega=\omega_{s}$ for the values $\left(V_{i}, \phi_{i}, \omega_{s}\right)$ which, in general, will be different from those of the individual freerunning oscillators (when isolated from the system).

Now the transient of the coupled-oscillator system will be addressed. During this transient the amplitude and phase of each oscillator are time varying. The corresponding system is derived from (1) by modifying the frequency variable to the form $\omega \rightarrow \omega_{o}+D_{t} / j$ [16], where $D_{t}$ is the time-derivative operator and $\omega_{o}$ is the free-running frequency of one of the oscillators in the array, which is arbitrarily taken as the fundamental frequency of the Fourier basis. Thus, we obtain the following system of nonlinear differential equations:

$$
\begin{aligned}
& Y_{i}\left(V_{i}(t), \omega_{o}+\frac{D_{t}}{j}\right) X_{i}(t)+\sum_{k=1}^{N} Y_{i k}^{c}\left(\omega_{o}+\frac{D_{t}}{j}\right) X_{k}(t)=0, \\
& i=1, \ldots, N
\end{aligned}
$$

We emphasize that unlike the analyses [9]-[11], the nonlinear admittance functions $Y_{i}\left(V_{i}, \omega\right)$ in (2) are not linearized about the locked steady state. On the contrary, the nonlinear dependence of these functions on the amplitude variable will be used to predict the whole transient dynamics.

During the transient state, the time variation of the phasors $X_{i}(t)$ is in general much slower than the time scale provided by the oscillation frequency $\omega_{o}$. Then, these components are narrowband signals that can be expressed as:

$$
X_{i}(t)=\int_{-\omega_{b}}^{\omega_{b}} X_{i}(\omega) e^{j \omega t} d \omega, \quad i=1, \ldots, N
$$

where $\omega_{b}$ is the maximum bandwidth of the time-varying phasors. Using this result, the dependence of the admittance functions on the complex frequency can be approached by a first-order Taylor series, providing:

$$
a_{0}^{i}\left(V_{i}\right) X_{i}(t)+a_{1}^{i}\left(V_{i}\right) \dot{X}_{i}(t)+\sum_{k=1}^{N} Y_{i k}^{c}\left(\omega_{o}\right) X_{k}(t)=0
$$

where:

$$
a_{0}^{i}\left(V_{i}\right) \equiv Y_{i}\left(V_{i}, \omega_{o}\right), \quad a_{1}^{i}\left(V_{i}\right) \equiv-j \frac{\partial Y_{i}\left(V_{i}, \omega_{o}\right)}{\partial \omega}
$$

for $i=1, \ldots, N$. Note that once the nonlinear admittance functions $a_{0}^{i}\left(V_{i}\right)$ are available the derivatives $a_{1}^{i}\left(V_{i}\right)$ are extracted through finite differences in a straight-forward manner [14]. On the other hand, the frequency derivatives of the linear coupling network admittance matrix have been neglected, since these components remain nearly constant in the frequency range $\left[\omega_{o}-\omega_{b}, \omega_{o}+\omega_{b}\right]$. System (4) provides for the first time to our knowledge a set of nonlinear ODEs describing the transient behavior of a multi-oscillator system. In order to better understand the system dynamics, it is more illustrative to express the equation of each oscillator in (4) in terms of the phase and amplitude variables:

$$
\begin{aligned}
& a_{0}^{i}\left(V_{i}\right) V_{i}(t)+a_{1}^{i}\left(V_{i}\right)\left(\dot{V}_{i}(t)+j V_{i}(t) \dot{\phi}_{i}(t)\right)+ \\
& +\sum_{k=1}^{N} Y_{i k}^{c}\left(\omega_{o}\right) V_{k}(t) e^{j\left(\phi_{k}(t)-\phi_{i}(t)\right)}=0
\end{aligned}
$$

for $i=1, \ldots, N$. Each equation depends nonlinearly on the voltage amplitudes and phases through the functions $a_{0,1}^{i}\left(V_{i}\right)$ and the exponential components, respectively. Splitting each complex equation in (6) into real and imaginary parts, the whole system can be rewritten in compact form as:

$$
\dot{\bar{y}}(t)=f(\bar{V}(t), \bar{\phi}(t)), \quad \bar{y}=\left(\begin{array}{l}
\bar{V} \\
\bar{\phi}
\end{array}\right)
$$

where the set of state variables is composed by the components in the vectors $\bar{V}=\left(V_{1}, \ldots, V_{N}\right)^{t}$ and $\bar{\phi}=\left(\phi_{1}, \ldots, \phi_{N}\right)^{t}$. From (6), it can be straightforwardly derived that:

$$
f\left(\bar{V}(t), \bar{\phi}_{\alpha}(t)\right)=f(\bar{V}(t), \bar{\phi}(t)), \quad \forall \alpha \in \mathcal{R}
$$

where $\bar{\phi}_{\alpha}(t) \equiv\left(\phi_{1}+\alpha, \ldots, \phi_{N}+\alpha\right)^{t}$. This property shows that system (6) remains invariant under a global constant phase shift. Then, at any time, the transient trajectories of the phase variables $\phi_{i}(t)$ will contain a constant component that depends on the chosen initial conditions. Expressing the first harmonic in terms of the phase and amplitude variables as $V_{i}(t) e^{j\left(\omega_{o} t+\phi_{i}(t)\right)}$, it is seen that the instantaneous frequency of each $i$-th oscillator is $\omega_{o}+\dot{\phi}_{i}(t)$.

Once the functions $a_{0}^{i}\left(V_{i}\right)$ and $a_{1}^{i}\left(V_{i}\right)$ of each $i$-th oscillator have been identified [14], the transient trajectories of the amplitude and phase of each oscillator of the array can be simulated through time-integration of the nonlinear system of ODEs (7).

\section{APPLICATION TO A SYSTEM OF COUPLED OSCILLATORS}

The previous analysis will be illustrated through its application to a system of $N=3$ coupled oscillators, illustrated in Fig. 1. The oscillators are coupled through resistively loaded microstrip lines (width $=0.28 \mathrm{~mm}$, length $=$ $33.9 \mathrm{~mm}$ ). The admittance matrix of the $N$-port coupling network is calculated in commercial software, providing the coupling terms $Y_{i k}^{c}\left(\omega_{o}\right)$. Note that since the array is unidimensional, the coupling terms of not-adjacent ports vanish, obtaining $Y_{i k}^{c}\left(\omega_{o}\right)=0$ for $|i-k|>1$. As a result, the KCL equation of each oscillator in (6) is explicitly coupled only to the adjacent ones. In the first place, the technique will be tested by its application to a system of $N=3$ Van der Pol type oscillators. Then, the technique will be applied to a realistic system of $N=3$ coupled FET-based oscillators.

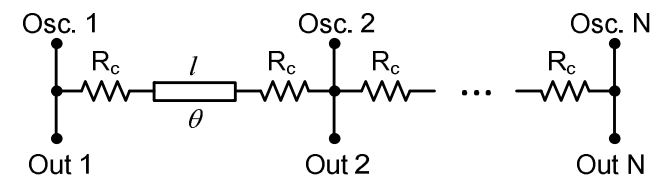

Fig. 1. Schematic of the system of $N$ coupled oscillators. 


\section{A. System of $N=3$ coupled Van der Pol type oscillators}

The schematic of each oscillator is shown in Fig. 2. It is composed of an RLC resonator in parallel with a nonlinear voltage-controlled current source presenting negative resistance for small-signal amplitude.

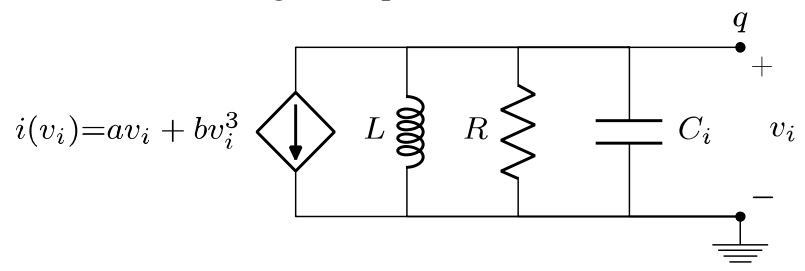

Fig. 2. Schematic of the Van der Pol oscillator. The circuit parameter values are $a=-0.03 \Omega^{-1}, b=0.01 \mathrm{~A} / \mathrm{V}^{3}, L=1 \mathrm{nH}, R=50 \Omega$

The free-running frequency of each $i$-th oscillator can be tuned by means of the capacitor $C_{i}$. Two qualitatively different cases have been considered. In the first case, the capacitances of the three oscillators have been set to the same value: $C_{i}=10$ $\mathrm{pF}, i=1,2,3$, corresponding to the free-running frequency $\omega_{o} \approx 2 \pi \cdot 1.6 \mathrm{GHz}$. Therefore, the functions $a_{0}^{i}\left(V_{i}\right)$ and $a_{1}^{i}\left(V_{i}\right)$ are identical for the three oscillators. These functions have been identified through $\mathrm{HB}$ simulations of an isolated oscillator, following the procedure described in [14]. Then, the transient trajectories of the amplitude and phase variables of each oscillator have been calculated by integrating system (7). The initial conditions for the amplitude and phase variables have been respectively set near zero $\left(V_{i}=0.01 V, i=1,2,3\right)$, and zero $\left(\phi_{i}=0, i=1,2,3\right)$. The simulation results are shown in Fig. 3. As can be seen, the amplitude and phase trajectories go through a transient state of duration $t_{s} \approx 30 \mathrm{~ns}$. Once the steady state is reached, the amplitude components become constant, whereas the time variation of the phase components present the same constant slope $\dot{\phi}_{1}=\dot{\phi}_{2}=\dot{\phi}_{3}=\Delta \omega$, providing a common instantaneous frequency $\omega_{o}+\Delta \omega$. This scenario corresponds to a locked solution at the frequency $\omega_{s}=$ $\omega_{o}+\Delta \omega$. In the same figures, the simulation of the coupled system using the envelope transient technique with 7 harmonics in commercial software has been superimposed, showing very good agreement.

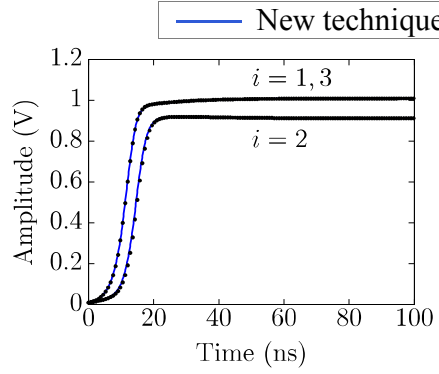

(a)

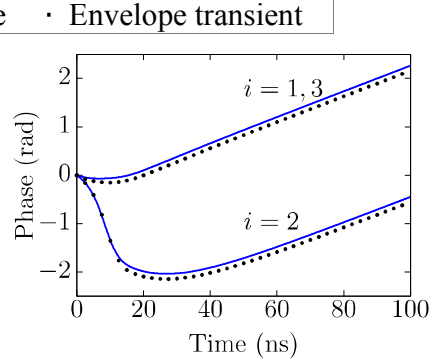

(b)
Fig. 3. System of three coupled Van der Pol oscillators. Locked case, with $C_{i}=$ $10 \mathrm{pF}, i=1,2,3$. (a) Amplitude trajectories. (b) Phase trajectories

In the second case, the capacitances of the three oscillators have been set to $C_{1,3}=11 \mathrm{pF}, C_{2}=10 \mathrm{pF}$. As can be seen in Fig. 4(b), in this case the instantaneous frequencies of the outermost oscillators $\omega_{o}+\dot{\phi}_{1,3}$ are different from the central one $\omega_{o}+\dot{\phi}_{2}$. Then, this configuration leads the system to an unlocked state. The different oscillation frequencies present in the coupled systems make the amplitude and phase variables exhibit an oscillatory behavior [see Fig. 4(a)]. As in the previous case, the envelope transient simulation results have been superimposed.

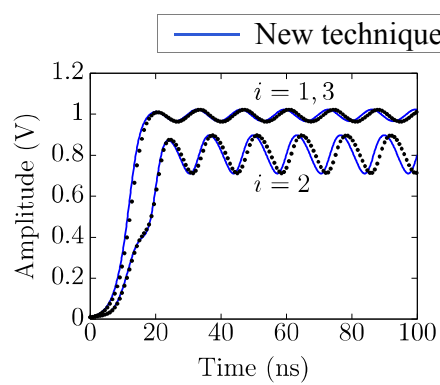

(a)

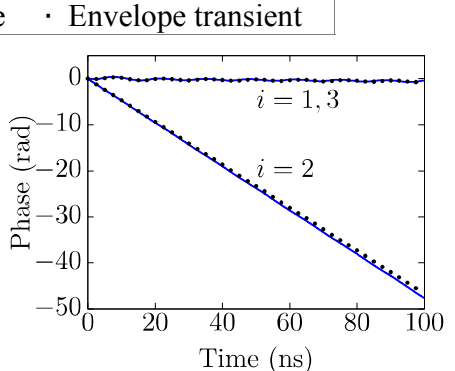

(b)
Fig. 4. System of three coupled Van der Pol oscillators. Unlocked case, with $C_{1,3}=11 \mathrm{pF}, C_{2}=10 \mathrm{pF}$. (a) Amplitude trajectories (b) Phase trajectories.

\section{B. System of $N=3$ coupled FET oscillators.}

The oscillators are designed using a field-effect transistor (NE3210S01) on a RO4003C substrate. The schematic of the single oscillator is shown in Fig. 5. A varactor diode (SMV1233) is used as a tuning element with the dc voltage $\eta_{i}$, $i=1,2,3$. The observation node $q$ of each oscillator agrees with the output node, where a $50 \Omega$ resistance is connected in parallel with the coupling network.

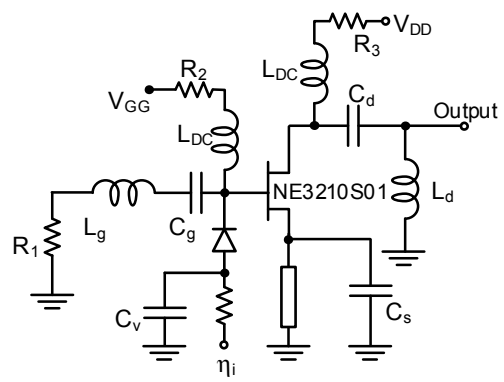

Fig. 5. Schematic of the FET-based oscillator.

In the first case, the tuning values $\eta_{1,3}=2.1 \mathrm{~V}, \eta_{2}=2 \mathrm{~V}$ have been used. The initial conditions for the amplitude and phase variables have been respectively set to $V_{i}=1 \mathrm{mV}$, and $\phi_{i}=0$, for $i=1,2,3$. The transient evolution to the steady state of the output voltage amplitudes of the three oscillators, calculated through system (7), are shown in Fig. 6(a). As can be seen, the system evolves towards a locked state after a transient of duration $t_{s} \approx 14 \mathrm{~ns}$, where the amplitude variables become constant. As in the previous analysis, the results have been validated with the envelope transient technique.

In the second analysis, the three tuning voltages have been equated to the value $\eta_{1,2,3}=2 \mathrm{~V}$. The simulation results are shown in Fig. 6(b), where an unlocked state is observed. The transient duration is similar to the one in the locked case, and the $y$-scale has been reduced to show the small-amplitude oscillatory behavior. In this case, both the new technique and the envelope transient show a similar qualitative behavior, 
although there exist quantitative discrepancies due to the high harmonic component of the unlocked solution.

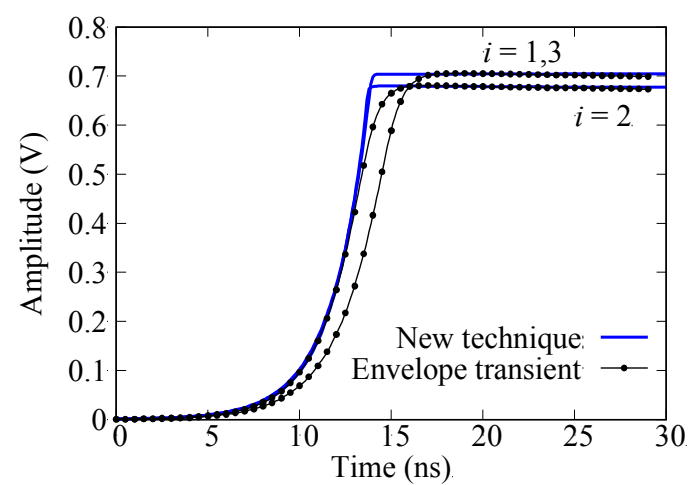

(a)

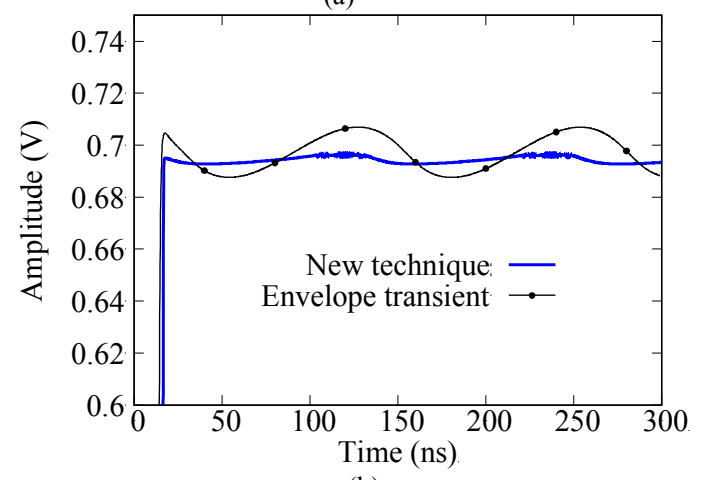

(b)

Fig. 6. System of three coupled FET oscillators. Comparison of the simulated amplitude trajectories with those of the envelope-transient simulation. (a) Locked case, $\eta_{1,3}=2.1 \mathrm{~V}, \eta_{2}=2 \mathrm{~V}$. (b) Unlocked case, $\eta_{1}=\eta_{2}=\eta_{3}=2 \mathrm{~V}$.

Finally, the transient trajectory of the amplitude of the oscillator $i=1$ has been measured when evolving to the locked case of Fig. 6(a). For this purpose, the oscillator output signal is connected to a Keysight Infiniium 90804A oscilloscope. The oscillation start-up is triggered at $t=0$ by an ON/OFF signal applied to the drain bias voltage of the oscillators. The measured transient to the locked state is compared in Fig. 7 with the amplitude $V_{1}(t)$ resulting from the simulation of system (7) and the envelope transient results. As can be seen, the new technique is able to approach the length and the asymptotic component of the transient trajectory.

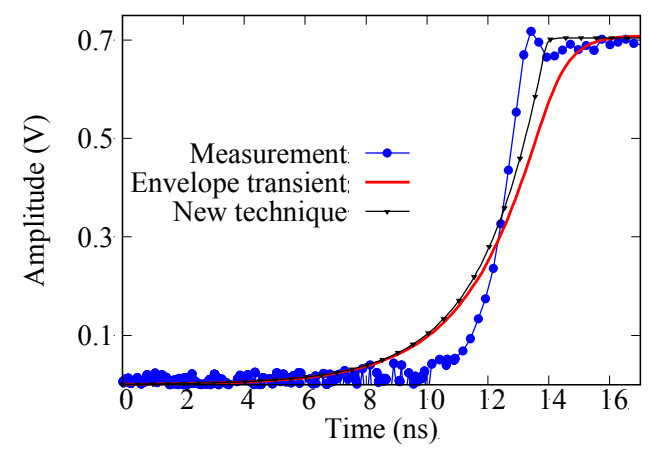

Fig. 7. System of three coupled FET oscillators. Measured output voltage of the oscillator $i=1$ when evolving to the locked case, with $\eta_{1,3}=2.1 \mathrm{~V}, \eta_{2}=$ $2 \mathrm{~V}$. The amplitude transient trajectory simulated with system (7) and with the envelope transient technique is superimposed.

\section{CONCLUSION}

A realistic formulation to predict the transient behavior of systems containing oscillators has been presented. The formulation relies on nonlinear reduced-order models of the individual oscillators. These models are derived from a nonlinear admittance function extracted from harmonic balance simulations. The formulation has been illustrated through its application to a system of three coupled oscillators. Results have been successfully compared with circuit-level envelopetransient simulation - which will fail or be impractical under a large number of elements - as well as with measurements.

\section{ACKNOWLEDGMENT}

This work was supported by the Spanish Ministry of Science, Innovation and Universities and the European Regional Development Fund (ERDF/FEDER) under the research project TEC2017-88242-C3-1-R.

\section{REFERENCES}

[1] N. B. Buchanan and V. Fusco, "Single VCO chipless RFID near-field reader," Electron. Lett., vol. 52, no. 23, pp. 1958-1960, Nov. 2016.

[2] J. J. Lynch and R. A. York, "Synchronization of oscillators coupled through narrow-band networks," IEEE Trans. Microw. Theory Tech., vol. 49, no. 2, pp. 237-249, 2001.

[3] T. Heath, "Simultaneous beam steering and null formation with coupled, nonlinear oscillator arrays," IEEE Trans. Antennas Propag., vol. 53, no. 6, pp. 2031-2035, 2005.

[4] R. J. Pogorzelski, "Continuum modeling of coupled oscillator arrays with coupling delay," Radio Sci., vol. 43, no. 4, p. n/a-n/a, Aug. 2008.

[5] R. Moussounda, "Analysis and design of coupled-oscillator arrays for microwave systems," The Ohio State University, 2014.

[6] T. Kijsanayotin, J. Li, and J. F. Buckwalter, "A 70-GHz LO PhaseShifting Bidirectional Frontend Using Linear Coupled Oscillators," IEEE Trans. Microw. Theory Tech., vol. 65, no. 3, pp. 892-904, Mar. 2017.

[7] L. Qi, Q. Xie, L. Deng, and Z. Wang, "A novel terahertz phased array based on coupled oscillators," in 2018 IEEE MTT-S International Wireless Symposium (IWS), 2018, pp. 1-3.

[8] A. Suárez, A. Collado, and F. Ramírez, "Harmonic-balance techniques for the design of coupled-oscillator systems in both unforced and injection-locked operation," IEEE MTT-S Int. Microw. Symp. Dig., vol. 2005, pp. 887-890, 2005.

[9] A. Suarez, S. Sancho, and F. Ramirez, "General formulation for the analysis of injection-locked coupled-oscillator systems," IEEE Trans. Microw. Theory Tech., vol. 61, no. 12, pp. 4730-4744, Dec. 2013.

[10] A. Suárez, F. Ramírez, and S. Sancho, "Stability and noise analysis of coupled-oscillator systems," IEEE Trans. Microw. Theory Tech., vol. 59, no. 4 PART 1, pp. 1032-1046, Apr. 2011.

[11] A. Suárez, F. Ramírez, S. Sancho, and J. M. Collantes, "Global Stability Analysis of Coupled-Oscillator Systems," IEEE Trans. Microw. Theory Tech., vol. 63, no. 1, pp. 165-180, 2015.

[12] A. Suarez, Analysis and Design of Autonomous Microwave Circuits. Wiley, 2009.

[13] A. Suarez, S. Sancho, and F. Ramirez, "Growth-rate function for the nonlinear analysis of the transient dynamics of microwave oscillators," in IEEE MTT-S International Microwave Symposium Digest, 2016, pp. $1-4$.

[14] S. Sancho, A. Suarez, F. Ramirez, and M. Ponton, "Analysis of the Transient Dynamics of Microwave Oscillators," IEEE Trans. Microw. Theory Tech., vol. 67, no. 9, pp. 3562-3574, Sep. 2019.

[15] F. Ramírez, A. Suárez, and S. Sancho, "Harmonic-balance technique for the shortening of the initial transient of microwave oscillators," in IEEE MTT-S International Microwave Symposium Digest, 2005, vol. 2005, pp. 805-808.

[16] K. Kurokawa, "Some basic characteristics of broadband negative resistance oscillator circuits," Bell Syst. Tech. J., vol. 48, no. 6, pp. 19371955, Jul. 1969. 